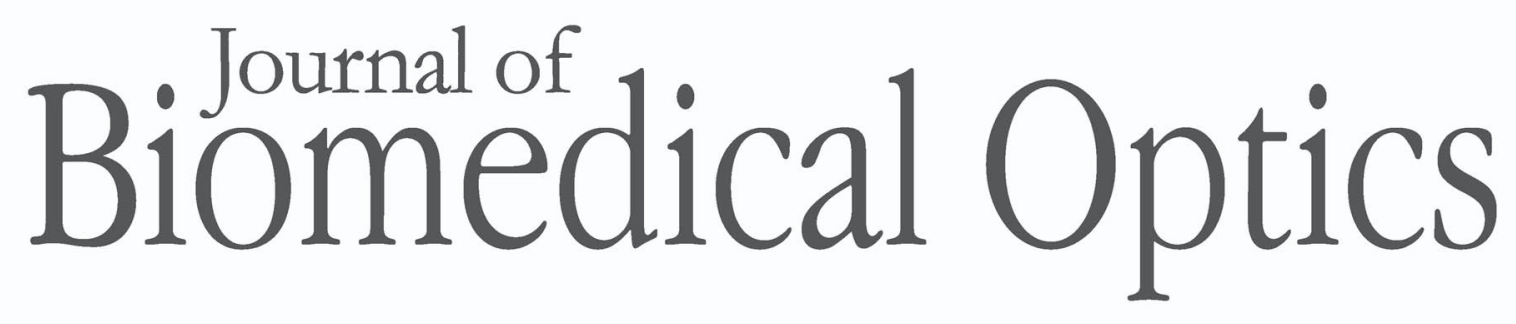

\title{
Simultaneous fluorescence and positron emission tomography for in vivo imaging of small animals
}

Bin Zhang

Shuangquan Liu

Fei Liu

Xiaochun Zhang

Yanyan $\mathrm{Xu}$

Jianwen Luo

Baoci Shan

Jing Bai 


\section{Simultaneous fluorescence and positron emission tomography for in vivo imaging of small animals}

\author{
Bin Zhang, ${ }^{a}$ Shuangquan Liu, ${ }^{b}$ Fei Liu, ${ }^{a}$ Xiaochun Zhang, ${ }^{c}$ \\ Yanyan Xu, ${ }^{a}$ Jianwen Luo, ${ }^{d}$ Baoci Shan, ${ }^{b}$ and Jing Bai ${ }^{a}$ \\ aTsinghua University, Department of Biomedical Engineering, Beijing, \\ 100084, China \\ ${ }^{b}$ Chinese Academy of Sciences, Institute of High Energy Physics, Key \\ Laboratory of Nuclear Analytical Techniques, Beijing, 100049, China \\ 'XinAoMDT Technology Co., Ltd., Langfang, 065001, China \\ ${ }^{\mathrm{d}}$ Tsinghua University, Center for Biomedical Imaging Research, \\ Beijing, 100084, China
}

Abstract. Simultaneous positron emission tomography (PET) and fluorescence tomography (FT) for in vivo imaging of small animals is proposed by a dual-modality system. This system combines a charge-coupled device-based nearinfrared fluorescence imaging with a planar detector pairbased PET. With $\left[{ }^{18} \mathrm{~F}\right]$-2-fluoro-2-deoxy-d-glucose radioactive tracer and the protease activated fluorescence probe, on the one hand, the simultaneous metabolic activity and protease activity in tumor region are revealed by the PET and $\mathrm{FT}$, respectively. On the other hand, the protease activity both on the surface layer and the deep tissue of the tumor is provided by the fluorescence reflection imaging and FT, respectively. (C) 2011 Society of Photo-Optical Instrumentation Engineers (SPIE). [DOI: 10.1117/1.3665438]

Keywords: fluorescence imaging; multimodality imaging system; positron emission tomography.

Paper 11564LR received Oct. 1, 2011; revised manuscript received Nov. 7, 2011; accepted for publication Nov. 14, 2011; published online Dec. 5, 2011.

A multimodality system for in vivo imaging of small animals has become an important tool for modern biomedical research, as it offers advantages of combining the complementary characteristics of different modalities. Representative examples of a multimodality system are the combination of a high spatial resolution anatomical imaging modality and a functional imaging modality, such as computed tomography (CT) or magnetic resonance imaging (MRI), and positron emission tomography (PET) or single photon emission computed tomography. Meanwhile, multimodality imaging systems, which combine fluorescence imaging with CT or MRI, have also been developed.

The hybrid of two functional modalities, PET and fluorescence imaging, is also interesting and scientifically attractive. ${ }^{1}$ This type of dual-modality system has several advantages. ${ }^{1-3}$ First, different functional information can be simultaneously visualized. For example, with $\left[{ }^{18} \mathrm{~F}\right]$-2-fluoro-2-deoxy-d-glucose $\left({ }^{18} \mathrm{~F}-\mathrm{FDG}\right)$ and enzyme activated fluorescence probe, both

Address all correspondence to: Jing Bai, Tsinghua University, Department of Biomedical Engineering, Heqing Road, Haidian District, Beijing, 100084 China; Tel: 86-10-62786480; E-mail: deabj@tsinghua.edu.cn. metabolic activity and protease activity can be visualized at the same time in the tumor. Second, the co-registration of PET and fluorescence images is very convenient in the hybrid system. Since the imaging object is scanned in the same posture, no specific fusion approaches are needed. Finally, with specific probes which incorporate a fluorescent optical marker and radiotracer into a single molecule, ${ }^{4-6}$ a hybrid PET and fluorescence system allows a simultaneous description of the characteristics of these new molecular imaging probes.

Several imaging systems which combine fluorescence and radionuclide have been developed in recent years. ${ }^{2,3,7-9}$ Compared with these systems, our dual-modality system employed an electron multiplying charge-coupled device and was more sensitive. ${ }^{10}$ Besides, it can provide noncontact and full-angle fluorescence tomography without PET priors. However, the dynamic data acquisition strategy employed in our previous work will degrade the reconstruction results when the integration time is more than $5 \mathrm{~s}$.

In this work, we expanded the function of the dual-modality system which can provide not only PET and fluorescence tomography (FT) but also fluorescence reflection imaging (FRI). The PET and fluorescence data were acquired in a step mode rather than a dynamic acquisition mode, hence improving the quality of the reconstruction results. With ${ }^{18}$ F-FDG radioactive tracer and the protease activated fluorescence probe, the simultaneous protease activity and the metabolic activity in a tumor region are revealed by the PET and FT, respectively. Additionally, the fluorescence distribution on the surface layer and the deep tissue of the tumor is provided by FRI and FT, respectively.

The structure of the system, including schematic and photograph, is shown in Fig. 1 and is similar with our previous system. ${ }^{10}$ Two broad-beam illumination sources were added for fluorescence reflection imaging, and a mode switcher was employed to switch between FT and FRI mode. Additionally, a $35 \mathrm{~mm}$ f/1.6 lens (C3514-M, Pentax, Japan) was used to enlarge the field of view of fluorescence imaging.

In vivo imaging of a tumor bearing nude mouse was performed to test the simultaneous performance of the PET and fluorescence dual-modality system. ${ }^{18}$ F-FDG was used as the radioactive tracer for PET imaging. A protease activated fluorescence probe (Prosense 750, PerkinElmer, Massachusetts) was used for fluorescence imaging, which becomes highly fluorescent on interaction with cathepsins such as cathepsin B, L, and $\mathrm{S}$. Therefore, by using a fluorescent probe sensitive to cathepsins which are overexpressed in a variety of malignant tumors, visualization of biochemical abnormalities in the tumor could be achieved.

About $1 \times 10^{6} 4 \mathrm{~T} 1$ mouse breast tumor cells (Cell Bank of the Chinese Academy of Sciences, Shanghai, China) were injected into the right axilla of a BALB/c nude mouse. Tumor growth was monitored and the mouse was imaged when the tumor was about $8 \mathrm{~mm}$ in diameter.

In this experiment, the PET and FT projections were acquired simultaneously. Two nmol $(150 \mu \mathrm{l})$ ProSense 750 was intravenously injected $24 \mathrm{~h}$ before imaging, as recommended by the description of product, and $1 \mathrm{mCi} 18 \mathrm{~F}-\mathrm{FDG}$ was intravenously

1083-3668/2011/16(12)/120511/3/\$25.00 @ 2011 SPIE 


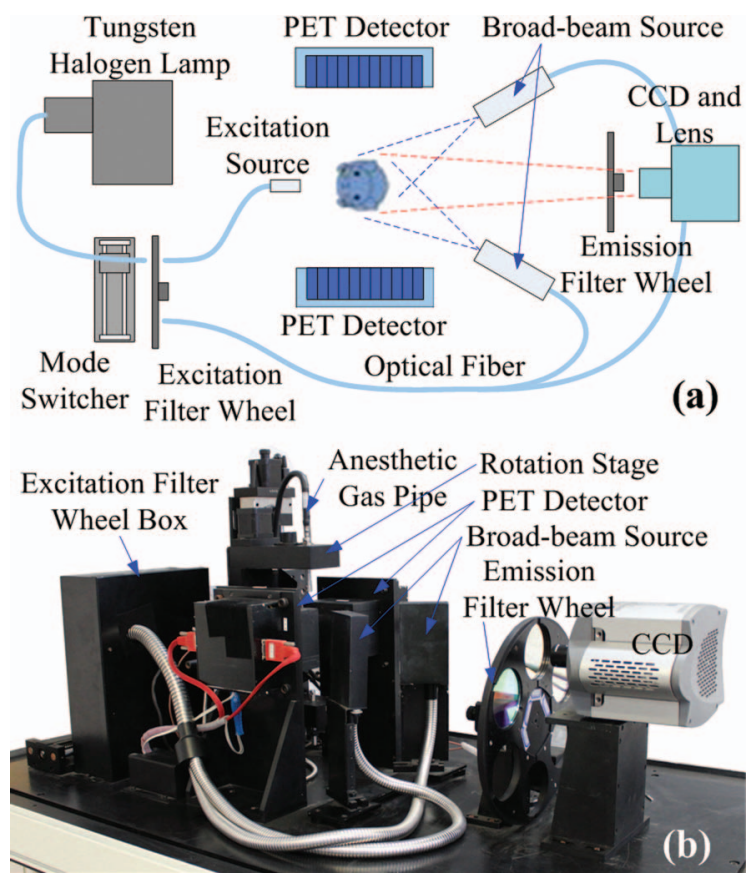

Fig. 1 Structure of the dual-modality system. (a) Schematic top view of the system. (b) Photo of the system.

injected $1.5 \mathrm{~h}$ before imaging. The fluorescent imaging was acquired using the $735 \mathrm{~nm}$ excitation filter (735AF50, Caliper Life Sciences, Massachusetts) and ICG emission filter (810-875 nm, Caliper Life Sciences, Massachusetts). The background images were acquired using the $680 \mathrm{~nm}$ excitation filter (735AF50, Caliper Life Sciences, Massachusetts). First, the mode switcher switched to the reflection mode and 64 reflection fluorescence images and 64 background images were sequentially acquired during the $360^{\circ}$ rotation both with $5 \mathrm{~s}$ exposure time. Second, the mode switcher switched to the transmission mode. Thirty-two PET projections and 64 fluorescence projections were acquired simultaneously during the $360^{\circ}$ rotation. The integration time of PET is $20 \mathrm{~s}$ and the exposure time of CCD is $5 \mathrm{~s}$. All the data were acquired in the step mode. Throughout imaging experiments, anesthesia was maintained with isoflurane-oxygen gas mixture. All animal experiments were conducted under the protocol approved by the Institutional Animal Care of Tsinghua University.

When the data acquisition was completed, the PET and fluorescence data were processed separately. ${ }^{10}$ The reflection fluorescence images were corrected by subtracting the background images excited by the background wavelength. ${ }^{11}$ The recon-
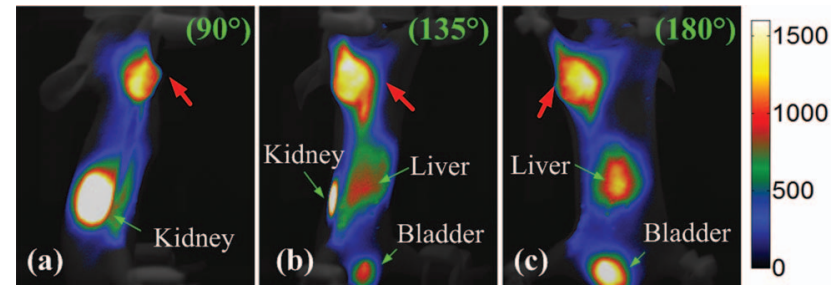

Fig. 2 Reflection fluorescence images of the tumor-bearing mouse at different angles. The tumor at the right axilla is indicated by arrows.
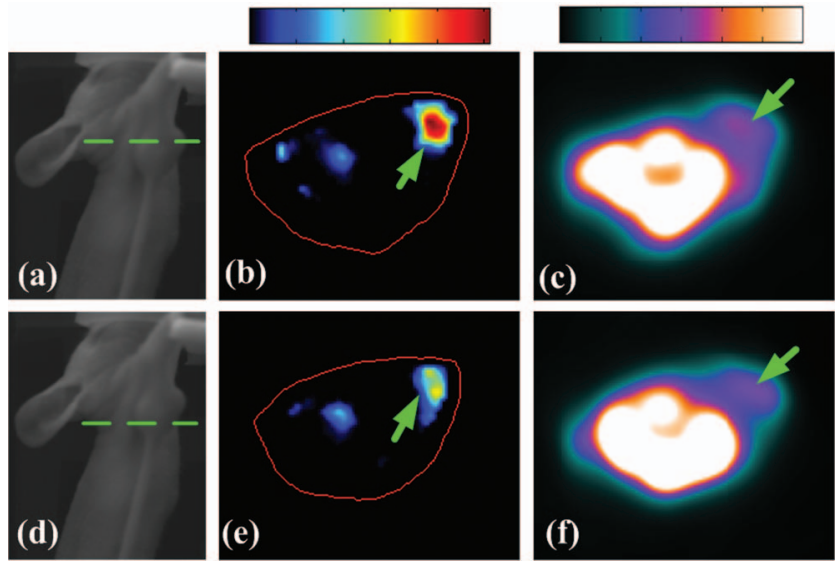

Fig. 3 Tomographic images of the tumor region. (a) and (d) The dashed lines indicate the different heights of the tomographic images. (b) and (e) Fluorescence tomographic images. The outer curve shows the surface of the mouse. (c) and ( $f$ ) The PET images. The arrows indicate the location of the tumor.

structed PET result had the dimensions of $128 \times 128 \times 128$ with a pixel size of $0.5 \times 0.5 \times 0.5 \mathrm{~mm}^{3}$. And in fluorescence reconstruction, only the tumor region was reconstructed and the reconstruction result had the dimensions of $31 \times 31 \times 18$ with a pixel size of $1 \times 1 \times 1 \mathrm{~mm}^{3}$.

The reflection fluorescence images of the tumor-bearing mouse are shown in Fig. 2. The fluorescence signals are in accordance with the tumor appearance on the white-light images.

The reconstructed PET and FT images are shown in Fig. 3. The FT images [Figs. 3(b) and 3(e)] reveal the distribution of the fluorescent probe in the tumor region. The PET images [Figs. 3(c) and 3(f)] reveal the metabolic activity in the tumor region. Both reflection fluorescence images (Fig. 2) and the FT images [Figs. 3(b) and 3(e)] reveal the accumulation of the fluorescence probes in the tumor region and indicate overexpressed cathepsins in the tumor region. Figure 4
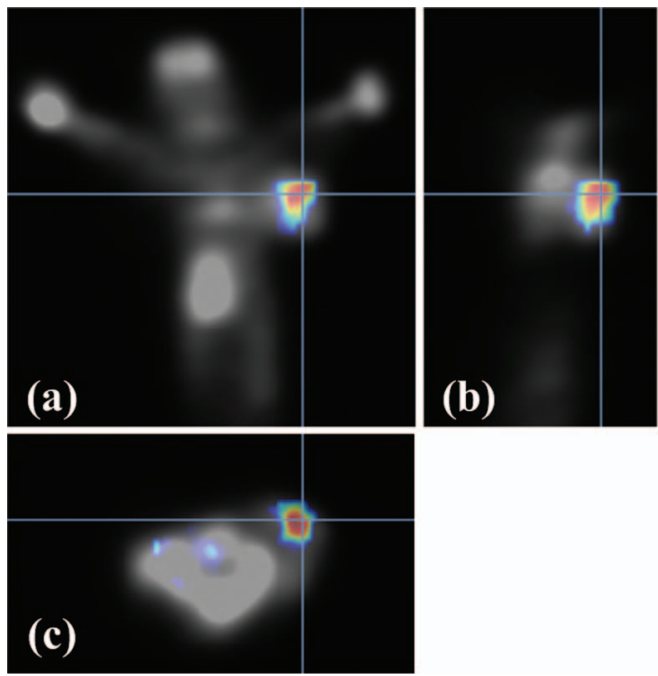

Fig. 4 Co-registration results of PET and FT images. The coronal plane (a), sagittal plane (b), and transverse plane (c) of the co-registration results in the tumor region. 
shows the co-registration of PET and FT images. The coregistered images simultaneously illustrate the uptake of ${ }^{18} \mathrm{~F}$ FDG and the fluorescence distribution in the inside of the tumor region.

Simultaneous in vivo visualization of biochemical abnormalities in tumor regions, such as cathepsin overexpression and metabolic activity, is very important in biomedical research. The information is revealed simultaneously by the proposed PET and fluorescence tomography dual-modality system for small animals in vivo. With the ${ }^{18} \mathrm{~F}-\mathrm{FDG}$ radioactive tracer and the cathepsin sensitive fluorescence probe, both the metabolic activity and the cathepsins overexpression in the tumor are revealed. Meanwhile, the system provides fluorescence distribution not only on the superficial layer but in the deep tissue of the tumor through reflection fluorescence imaging and fluorescence tomography, respectively. Additionally, by using the step mode in data acquisition, both the PET and FT results are greatly improved when the integration time is more than a few seconds.

Another application of the dual-modality system is to use the PET priors to guide the fluorescence reconstruction. ${ }^{2}$ Additionally, dual-labeled imaging agents which contain multilabeled probes in a single molecule, such as the fluorescence and nuclear dual-labeled agents, ${ }^{4-6}$ are better selections in simultaneous examination of biomarker clusters. We will adopt the dual-labeled imaging agents in our future work for simultaneous imaging.

\section{Acknowledgments}

The authors thank the reviewers for their helpful suggestions. This work was supported in part by the National Basic Research Program of China (973) under Grant No. 2011CB707701, the National Natural Science Foundation of China under Grant Nos. 81071191, 60831003, 30930092, and 30872633, the Beijing Natural Science Foundation under Grant No. 3111003, and the Tsinghua-Yue-Yuen Medical Science Foundation.

\section{References}

1. J. Culver, W. Akers, and S. Achilefu, "Multimodality molecular imaging with combined optical and SPECT/PET modalities," J. Nucl. Med. 49(2), 169-172 (2008).

2. C. Q. Li, G. Wang, J. Qi, and S. R. Cherry, "Three-dimensional fluorescence optical tomography in small-animal imaging using simultaneous positron-emission-tomography priors," Opt. Lett. 34(19), 2933-2935 (2009).

3. J. Peter, D. Unholtz, R. B. Schulz, J. Doll, and W. Semmler, "Development and Initial Results of a Tomographic Dual-Modality Positron/Optical Small Animal Imager," IEEE Trans. Nucl. Sci. 54(5), 1553-1560 (2007).

4. L. Sampath, S. Kwon, S. Ke, W. Wang, R. Schiff, M. E. Mawad, and E. M. Sevick-Muraca, "Dual-labeled trastuzumab-based imaging agent for the detection of human epidermal growth factor receptor 2 overexpression in breast cancer," J. Nucl. Med. 48(9), 1501-1510 (2007).

5. A. Azhdarinia, N. Wilganowski, H. Robinson, P. Ghosh, S. Kwon, Z. W. Lazard, A. R. Davis, E. Olmsted-Davis, and E. M. Sevick-Muraca, "Characterization of chemical, radiochemical and optical properties of a dual-labeled MMP-9 targeting peptide," Bioorg. Med. Chem. 19(12), 3769-3776 (2011)

6. M. Nahrendorf, E. Keliher, B. Marinelli, P. Waterman, P. F. Feruglio, L. Fexon, M. Pivovarov, F. K. Swirski, M. J. Pittet, C. Vinegoni, and R. Weissleder, "Hybrid PET-optical imaging using targeted probes," Proc. Natl. Acad. Sci. U.S.A. 107(17), 7910-7915 (2010).

7. M. Autiero, L. Celentano, R. Cozzolino, P. Laccetti, M. Marotta, G. Mettivier, M. C. Montesi, P. Riccio, G. Roberti, and P. Russo, "Experimental study on in vivo optical and radionuclide imaging in small animals," IEEE Trans. Nucl. Sci. 52(1), 205-209 (2005).

8. D. L. Prout, R. W. Silverman, and A. Chatziioannou, "Detector concept for OPET-A combined PET and optical imaging system," IEEE Trans. Nucl. Sci. 51(3), 752-756 (2004).

9. J. H. Jung, Y. Choi, K. J. Hong, B. J. Min, J. Y. Choi, Y. S. Choe, K. H. Lee, and B. T. Kim, "Development of a dual modality imaging system: a combined gamma camera and optical imager," Phys. Med. Biol. 54(14), $4547-4559$ (2009).

10. S. Liu, B. Zhang, X. Wang, L. Li, Y. Chen, X. Liu, F. Liu, B. Shan, and J. Bai, "A dual modality system for simultaneous fluorescence and positron emission tomography imaging of small animals," IEEE Trans. Nucl. Sci. 58(1), 51-57 (2011).

11. N. C. Deliolanis, T. Wurdinger, L. Pike, B. A. Tannous, X. O. Breakefield, R. Weissleder, and V. Ntziachristos, "In vivo tomographic imaging of red-shifted fluorescent proteins," Biomed. Opt. Express, 2(4), 887-900 (2011) 